\title{
La raison et la foi. Des philosophes Polonais rapprochent les deux domaines
}

\section{Reason and faith - Polish philosophers' attempts to reconcile both realms}

\begin{abstract}
This paper presents three attempts of grasping the relations between faith and reason made by $\mathrm{M}$. Heller, J. Tischner and K. Tarnowski. Reason and faith are two foundations for human desire to understand the world. Without them, no attempts to understand can take place. As Heller points out, both of them, due to cooperation, support development and help to eliminate dangerous simplifications. Faith, or more precisely - religious thinking - is, as J. Tischner famously argued, a condition for dialogue, for being open to others and community. Faith gives strength and courage, supports the search for existing (although very often unconscious) ties that bonds people. Faith allows us to discover the meaning and purpose of our lives. K. Tarnowski goes even further and claims, that faith reveals "the surplus" of meaning, which invites us to investigate what is impossible to express - the Mystery. Only in the face of Mystery, by a communion with the Other, can we feel that the presence of the Other is important for our existence. The three philosophers from Krakow emphasized in their works, that the relations between faith and reason, although connected and supplementing themselves, require much attention from both scientists, who create rational models of reality, and theologians, who aim to create a coherent vision linking these two aspects of human world.
\end{abstract}

\section{Keywords}

Science; religion; reason; theology; faith; Mystery.

Le désir de comprendre le monde et la soif de Dieu, sont les deux faces d'un même besoin primordial, que l'homme réalise ou plutôt qu'il essaye de réaliser et ceci depuis l'aube de son existence. Ce besoin essentiel de trouver le sens 
à sa propre existence ${ }^{1}$ tente de combler autant la religion que la philosophie. Ces domaines ne sont pas contradictoires et même, comme le précisait JeanPaul II, „,es deux ordres de connaissance mènent à la plénitude de la vérité"2.

Cependant, une involontaire opposition de la foi au savoir s'est implantée dans la conviction populaire. La foi diffère considérablement du savoir. Toutefois la ligne qui les sépare n'est pas précise et les relations entre les deux domaines restent difficiles à saisir de manière univoque. L'une de causes importantes de cet état de fait est, comme le remarque J. Tischner, la tension entre la raison et la foi, résultant du fait, que la réflexion sur les vérités révélées est une partie essentielle de l'acte de la foi.

Cette tension est due en grande partie aux limitations que nous imposent nos moyens d'expression disponibles, ainsi que nos moyens de compréhension, dans leur confrontation avec la richesse du contenu transcendant, fourni par la religion et par l'expérience religieuse. Cette tension devient souvent la source d'un conflit entre la raison et la foi, mais aussi, comme le souligne un autre philosophe de Cracovie M. Heller, ce ,conflit constitue une partie de l'acte de la foi”, suite à quoi „l'authenticité de la foi demande, d'accentuer ce conflit et non de le diluer par des pseudo explications"3.

\section{Désir de l'interaction}

Les démarches entreprises pour éclaircir les problèmes liés aux relations entre la raison et la foi sont importantes pour plusieurs raisons. L'une d'elles est signalée par Jean Paul II: „Il est essentiel que la raison d'une personne croyante, utilise sa faculté de réflexion pour la recherche de la vérité dans le cadre d'un processus qui va de la parole de Dieu vers une meilleure compréhension de cette parole"'s.

\footnotetext{
1 „Tout d'abord il faut citer le besoin de vivre le monde comme imprégné de sens, ce qui s'exprime en référence à la transcendance. Il n'est pas important à ce moment de préciser comment on conçoit cette transcendance - l'important est sa signification existentielle, car la nécessité de vivre le monde en tant qu'ayant du sens constitue la nécessité inaliénable existentielle de l'homme". J. A. Kłoczowski, Leszka Kołakowskiego myślenie o religii, en: Polacy o religii. Od myślenia religijnego do filozofii, J. Barcik, G. Chrzanowski (ed.), Kraków 2005, WN PAT, p. 288.

2 Jean-Paul II, Encyclique Fides et Ratio, no 34.

3 M. Heller, Nowa fizyka i nowa teologia, Tarnów 1992, Biblos, p. 58.

4 Jean-Paul II, Encyclique Fides et Ratio, no 73.
} 
Heller montre qu'une conciliation des deux domaines est possible si nous formulerions une hypothèse selon un certain sens du rationalisme du monde. Nous pouvons considérer, d'après A. Einstein, que ,... l'ensemble des impressions de nos sens est de telle sorte qu'elles peuvent être ordonnées par le raisonnement [...], c'est un fait, dont nous ne pouvons que nous étonner et que nous ne pourrons jamais comprendre". En acceptant ce point de vue, la théologie peut considérer que le rationalisme du monde ainsi que son existence sont les deux aspects de la création qui montrent que le rationalisme du Créateur se reflète dans la structure du monde. En se référant à Platon, certains admettent même que , «Dieu géométrise toujours» et, en conséquent, tout résultat de l'activité créatrice divine est géométrique (ou in extenso-mathématique) dans sa nature"s.

De telles hypothèses sont assez difficiles à justifier, mais des arguments „historiques” ainsi que „heuristiques” arguent en leur faveur. Nous pouvons remarquer, que l'acceptation du modèle rationnel du monde est un choix ayant les sources morales ${ }^{6}$. La décision de se laisser orienter toujours par ce qui est compréhensible, a été admis par notre culture tout en étant en quelque sorte un choix indépendant de nous-mêmes. Nous pouvons évidemment contester ce choix, toutefois nier la rationalité du monde parait être une entreprise aussi difficile que celle de la prouver. En particulier, comme l'indique M. Heller, „Alors, si notre étude rationnelle du monde donne des résultats si remarquables, visiblement notre choix de se laisser orienter par la rationalité est en quelque sorte conforme à la structure de l'univers. Le monde n'est pas un chaos irrationnel mais une réalité bien ordonnée. Ou autrement: la méthode rationnelle d'examiner le monde s'avère efficace dans son étude car le monde est imprégné de la raison. Cependant, il ne faut pas comprendre cette raison de manière anthropomorphique, comme quelque chose associée à la conscience humaine, mais comme cette faculté du monde de révéler sa structure, rationnellement ordonnée, sous réserve d'y appliquer les moyens de l'étude rationnelle"7.

Les sceptiques pourraient convaincre en se reportant aux critères qui nous permettent d'apprécier la valeur de l'hypothèse de la rationalité. Un tel critère,

5 M. Heller, O teologicznych interpretacjach fizycznych teorii stworzenia, en: StwórcaWszechświat-Człowiek. Wybór tekstów wygłoszonych podczas konferencji z cyklu Boże działanie w perspektywie nauki zorganizowanego przez Watykańskie Obserwatorium Astronomiczne (Specola Vaticana) $i$ Center for Theology and the Natural Sciences (CTNS; Berkeley, California): 1988-2001, 1 (2006), p. 199.

6 Voir M. Heller, Czy fizyka jest nauka humanistyczna??, Tarnów 1998, Biblos, p. 208.

7 M. Heller, Czy fizyka jest nauka humanistyczną?, p. 208. 
parlant en faveur de la vérification positive de l'hypothèse observée, considère sa valeur en fonction de sa fécondité heuristique. On peut indiquer les succès obtenus ainsi - en reculant dans l'antiquité et à son Logos - étant à la fois un principe raisonnable de l'ordre du monde et son âme. Cependant, plus frappant pour l'imagination de l'homme moderne, est le fait que depuis trois cent ans, le fondement du succès de la science est justement l'hypothèse de la structure rationnelle de l'univers dont le Mystère peut pénétrer l'esprit humain.

Dans la tradition chrétienne, la conviction de la rationalité du monde était si évidente, qu'elle n'exigeait aucune preuve. Le seul point de discorde demeurait de déterminer la relation entre la foi et la raison dans la découverte de ce monde. De plus, la forte relation réciproque entre les deux domaines n'était pas mise en doute. Dans l'époque prémoderne deux formules rivalisaient entre elles „la foi cherche la compréhension” ( fides quaerens intellectum) et „la compréhension cherche la foi" (intellectus quaerens fidem). Malgré que les deux paraissent contradictoires, cependant dans chacune d'elles ,la conviction du fait que l'harmonie de la foi religieuse et de la raison est possible, restait commune"s.

La formule „la foi cherche la compréhension” provient de la tradition platonicienne-augustine. Par contre, la conviction que ,la compréhension cherche la foi" est fondée sur le cercle de la pensée aristotélique-thomasienne. J. Tischner a remarqué que les deux formules sont l'expression de la pensée religieuse et que ,l'homme dont la raison recherche la foi et dont la foi recherche la raison, est une pensée religieuse (...). Et quoique le monde apparaisse différemment à la lumière de la raison et qu'à la lumière de la foi, la différence des apparences ne tue ni la foi ni la raison. Tout au contraire, une lumière est une tentation pour l'autre lumière, elle est une inspiration et une promesse tentante". Les deux formules soulignent l'activité de l'homme en quête de l'acquisition de la vérité - et il n'est pas important de considérer la foi ou le savoir comme primordiale; l'important est le fait de chercher.

Cette quête constitue l'essence de l'être; nous ne pouvons pas arrêter de chercher. Il n'est pas concevable que la foi serait indifférente au désir de comprendre, ni que la raison soit privée de la nostalgie de consolider ses réalisations dans les références ultimes. L'homme qui perd un de deux dimensions de cette relation auto-dépendante, tombe ,en danger de fragmentation interne de l'être, dans

8 J. Tischner, Wokół spraw wiary i rozumu, en: S. Wszołek (ed.), Refleksje na rozdrożu, Wybór tekstów z pogranicza wiedzy $i$ wiary, Tarnów 2000, Biblos, p. 15.

9 J. Tischner, Myślenie wedtug wartości, Kraków 1982, Znak, p. 339-340. 
une attitude de non-authenticité, dans une crise de sa propre identité"10. Sans les recherches qui se stimulent entre elles, la raison ainsi que la foi se meurent. Jusqu'à présent on a consacré beaucoup d'attention aux questions concernant les problèmes créés par la perte de la foi à cause de la raison. Nous n'allons pas inclure ce thème ${ }^{11}$. Ici nous évoquerons uniquement les effets négatifs de la trahison de la foi par la raison.

Le progrès scientifique et technologique qui s'est accompli au cours de derniers siècles, est sans contestation un succès pour la raison humaine. L'orgueil des savants formés en positivistes, a amené à répandre l'idée que la raison peut résoudre, par elle-même, les problèmes de l'humanité. Aujourd'hui ce point de vue est déjà assez rare - au moins parmi les scientifiques intégrant la réflexion sur les possibilités de l'esprit humain ou bien sur les limites méthodologiques de la science. Toutefois, de nombreux croyants et même des prêtres considèrent que la raison constitue une menace pour la foi. Ils en tirent la conclusion, que la foi peut et même doit se passer de l'appui de la raison. Pour les partisans d'une telle conviction, un croyant idéal est un homme qui ne poserait pas de questions, qui suivrait inconditionnellement les rituels imposés par la religion et obéirait aux consignes religieuses.

Cependant, une telle forme de religiosité est soumise à un nombre de dangers. Jean-Paul II a bien remarqué leur nocivité pour la foi: „Illusoire est la présomption que la foi peut avoir un impact plus fort, sur un esprit faible. Au contraire, elle est alors menacée d'un grave danger, car elle peut alors être ramenée au niveau du mythe ou bien de la superstition"12. Un manque de réflexion sur la foi mène très souvent à l'aplanissement de son plus profond contenu et même à l'omission ou sa transformation en de sa propre opposition. „Quand un théologien ne veut pas se servir de la philosophie, apparaît alors que la philosophie peut représenter inconsciemment le danger de se renfermer dans des structures mentales peu aptes à la compréhension de la foi"'13.

Un rejet ou la seule ignorance des exigences de la raison menacent l'autonomie de la foi, car cela l'expose à être la proie de toutes sortes des charlatans, démagogues, idéologues ou enfin à des hommes qui grâce à son aide essayent de réaliser leurs propres intérêts. Cela concerne autant les fidèles que les

\footnotetext{
${ }^{10} \mathrm{~J}$. Tischner, Myślenie wedtug wartości, p. 341.

${ }^{11}$ Jean-Paul II a consacré beaucoup d'attention à cette question, voir Fides et Ratio.

12 Jean-Paul II, Encyclique Fides et Ratio, no 48.

13 Jean-Paul II, Encyclique Fides et Ratio, no 77.
} 
prêtres. Ayant ceci en vue, Jean Paul II indiquait qu'un manque de soutien de la part de la philosophie dans les études théologiques devient „la cause d'une grave négligence, autant dans la formation des ecclésiastiques qu'au sein de la théologie elle-même. Comme par exemple: le manque d'intérêt pour la pensée et la culture modernes, qui mène à l'atrophie de quelconques tentatives au dialogue ou à l'approbation sans critique des concepts philosophiques"14. Dans le dernier exemple, il vaut rappeler la popularité dont jouissait le créationnisme - une théorie pseudo-scientifique rejetant la théorie de l'évolution - chez certains membres du clergé polonais et chez certains politiciens catholiques. Ce n'est qu'après de fortes protestations des scientifiques des écoles catholiques qu'on est arrivé à rejeter l'absurde projet d'introduire le créationnisme dans les programmes scolaires à place de la théorie de l'évolution.

Un manque de contact avec la pensée moderne entraîne une carence dans la compréhension de la réalité qui nous entoure et éveille un sentiment d'aliénation, et même de rejet par le monde incompris. Le sentiment d'un manque d'acceptation mène à la paranoïa; le monde est suspect, de même que ceux qui ont une pensée différente. Naît alors la tentation de prendre la revanche, „trahi à ses yeux, le traître s'inspire de la philosophie du combat: il prend la revanche sur le monde, cherchant à sa destruction. Mais avant tout, il veut se soumettre et s'asservir les autres"'15.

La foi laissée à elle-même se transforme souvent en orgueil - non contrôlée par la raison, elle laisse le croyant croire en l'infaillibilité de ses propres interprétations des écris religieux. Ceci amène très souvent à nier de nombreuses valeurs, contenues dans une religion donnée. Les conséquences plus sérieuses liées au sentiment précédemment évoqué, mènent au fanatisme et dans les cas extrêmes, même aux guerres des religions. Le fanatisme est probablement la pire forme à laquelle peut aboutir une vie religieuse et constitue le pire danger pour les sociétés modernes. C'est pourquoi, pour l'éliminer de la vie sociale il faudrait faire davantage attention à la corrélation mutuelle de la foi et de la raison.

\footnotetext{
${ }^{14}$ Jean-Paul II, Encyclique Fides et Ratio, no 62.

${ }^{15} \mathrm{~K}$. Tarnowski, Józefa Tischnera myślenie wokół zbawienia, en: Polacy o religii. Od myślenia religijnego do filozofii, p. 54 .
} 


\section{Tentatives pour surmonter - science et religion}

Les philosophes polonais sont conscients des menaces existantes, pour cela ils soulignent la nécessité d'un retour aux sources, de l'union de la foi et de la raison. Dans un article aussi bref, il est difficile de soulever tous les aspects et touts les concepts intéressants. Pour cette raison je me vais me concentrer sur une présentation plus détaillée de trois propositions qui, d'après moi, sont les plus intéressantes, notamment celles des professeurs de L'Université Pontificale Jean Paul II de Cracovie: Michał Heller, Józef Tischner et Karol Tarnowski.

Le problème essentiel posé par ces penseurs, concerne la question suivante: dans quelle mesure la philosophie et d'autres disciplines scientifiques peuventelles étudier la religion, tout en préservant leur autonomie propre, et en même temps s'enrichir mutuellement?

Dans ses considérations, $\mathrm{M}$. Heller se concentre sur les relations entre la science et la religion. En s'inspirant de Jean Paul II, il montre la nécessité d'entreprendre rapidement et de façon déterminée des actions de sorte que la théologie contemporaine assimile des acquis de la science ${ }^{16}$. Dans ses écrits il essaye de démontrer que „la science peut épurer la religion des erreurs et des superstitions; la religion peut libérer la science de l'idolâtrie et des faux absolus. Chacune d'elles peut introduire l'autre dans un monde plus vaste, le monde où les deux peuvent se développer" ${ }^{\prime 17}$.

Selon M. Heller, le mécanisme de la liaison de la vision scientifique du monde avec la théologie peut s'expliquer par leur immersion commune et historique dans la culture. Les résultats des études historiques en donnent la preuve, révélant des liaisons étroites entre sphères de la raison et de la foi ${ }^{18}$. Le contexte culturel contient la vision du monde en vigueur dans une époque donnée. Ce qui pose problème pour les théologiens, c'est la variation de la vision scientifique du monde. Certains se présentent comme des „convictions”, dépassant les conventions arrêtées par les canons méthodologiques de l'enseignement scientifique. C'est pourquoi, conscients des problèmes vécus

16 Voir M. Heller, Nowa fizyka i nowa teologia, p. 91.

17 S. Wszołek, Postanie Jana Pawła II do George'a Coyne'a na tle encykliki Fides et Ratio, en: J. Mączka (ed.), Wiara i nauka. Materiaty z sesji naukowej i dyskusji panelowej, Kraków 2010, Wydawnictwo UJ, p. 248.

${ }^{18}$ Il prouvait à cette occasion que, ,les relations ne se limitent pas uniquement au «contexte de la découverte» mais parfois elles vont très profondément jusqu'à la structure-même des théories scientifiques". M. Heller, Nowa fizyka i nowa teologia, p. 92-95. 
par la théologie s'inspirant de la vision aristotélique du monde, les théologiens modernes observent (et ils le devraient observer) une grande prudence dans l'assimilation de l'approche scientifique de la réalité, à la suite d'une mise en question de cette vision classique.

Cependant, comme le souligne Heller, „l'utilisation d'une vision du monde est inévitable. La vision du monde est présente non seulement dans les exposés qui la concernent directement, mais aussi dans les présupposés de nombreux exposés qui concernent des sujets tout à fait différents; en plus, elle est présente dans tout le climat culturel. Si un théologien utilise un langage moderne, il se peut que tôt ou tard, et même sans le savoir, il fera référence à la vision scientifique du monde de son époque" ${ }^{19}$. Plutôt que d'éviter de se reporter à la vision du monde élaborée par la science, il sera encouragé à condamner les visions vieillottes souvent incompréhensibles ou même ridicules ${ }^{20}$.

C'est pourquoi il n'y a pas d'autre issue, „que d'accepter, dans exercice de la théologie, la vision moderne du monde, toutefois en la mettant sous contrôle, c'est à dire en maintenant le contact avec les enseignements afin de pouvoir juger ce qui dans la nouvelle vision du monde est réellement issu de la science et d'évaluer le statut (est-ce une hypothèse, un élément d'une théorie, une observation bien fondée...) de ce qui est uniquement issu d'une concession envers la mode intellectuelle dominante"21.

Il faut également remarquer que la vision scientifique du monde, quoique par principe elle est neutre quant aux questions éthiques et religieuses, génère cependant les conditions pour l'émergence de nouvelles valeurs morales. Elle constitue aussi un point de départ pour les débats sur les valeurs et contient en soi un indéniable aspect moral, dû également aux décisions des scientifiques qui dépassent la vision scientifique du monde. C'est pourquoi, tant que la vision scientifique du monde reste dans les limites abstraites du langage et de la structure, avec son horizon ouvert, elle est obligée „étant donné ses présupposés, à se poser des questions sur l'homme (...). Cette image crée un climat favorable à la reconnaissance de Dieu comme Raison, dont la manifestation est une réalité

${ }^{19}$ M. Heller, Naukowy obraz świata a zadania teologa, en: S. Wszołek (ed.), p. 226-228.

${ }^{20}$ Voir M. Heller, Naukowy obraz świata a zadania teologa, p. 226-228. „Déjà St. Augustin disait que si un chrétien montre son ignorance dans le domaine de la science et de la philosophie, il ridiculise sa religion aux yeux des païens". M. Heller, Naukowy obraz świata a zadania teologa, p. 227.

${ }^{21}$ M. Heller, Naukowy obraz świata a zadania teologa, p. 227. 
étudiée par la science"22. Le théologien doit cependant être conscient du fait que la représentation scientifique du monde est un produit des hommes, qu'elle est pour eux une chose proche, car ils participent à sa création. Pour cette raison il leur est souvent difficile d'accepter d'autres visions, y compris les essaies de description du monde des religions ${ }^{23}$.

Pour beaucoup de personnes, et aussi pour une grande partie des scientifiques, il est évident que les recherches en sciences humaines, se réfèrent à de multiples ressources, y compris des écris religieux. La réalité transcendante est inaccessible pour la science et sa réalité dépasse les compétences des scientifiques. D'autre part cependant, „les distinctions méthodologiques sur lesquelles s'appuie l'actuelle séparation des compétences des sphères de la foi et de la science, peuvent se montrer insuffisantes au fur et à mesure que leur évolution s'impose". Pour cette raison il n'est pas exclu dans l'avenir que la collaboration avec les scientifiques mènera à „la re-interprétation de certaines thèses de la foi" ${ }^{24}$. Cette collaboration, d'après Heller, peut se montrer bénéfique pour la théologie et la religion en général en évitant qu'un anthropomorphisme abusif fausse la perception du contenu de la foi mais au contraire favorise une relecture meilleure et plus précise.

Actuellement, dans le même esprit, d'autres philosophes commencent à se prononcer. Je cite un fragment d'un écrit de S. Wszołek, pouvant facilement illustrer les opinions de M. Heller: „Une théologie vivante doit prendre en considération les données des sciences et de la philosophie. La science apparaît comme une des loci theologici privilégiés. Il ne s'agit pas que la théologie accepte toutes les théories scientifiques, même bien enracinés, comme base d'une reformulation des concepts d'ordre religieux ... Un nombre des sphères de la réflexion humaine bien disciplinée (les sciences naturelles ne font pas exception) mènent vers les questions qui ne trouvent pas de réponse satisfaisante sur base des procédures spécifiques de la recherche et demandent une méta-discipline capable de les traiter de façon adéquate. La théologie, et plus précisément les théologiens, peuvent remplir ce rôle à condition de se donner la peine de comprendre les réalisations de la science. Par contre, la science ou plutôt les scientifiques peuvent gagner en s'intéressant à la théologie. Une telle ouverture de la science par rapport à la théologie ne vise pas à combler les carences de

\footnotetext{
${ }^{22}$ M. Heller, Nowa fizyka i nowa teologia, p. 67-68.

${ }^{23}$ M. Heller, Nowa fizyka i nowa teologia, p. 67-68.

${ }^{24}$ M. Heller, Nowa fizyka i nowa teologia, p. 70-71.
} 
notre connaissance de Dieu. Mais une pareille ouverture peut faire prendre conscience que certains problèmes et questions ne peuvent trouver une réponse satisfaisante en se référant uniquement à des disciplines diversifiées soumises à un dialogue interdisciplinaire" 25 .

Les relations entre la science moderne et la foi, plongées dans la même réalité culturelle déjà subissent une contrainte ${ }^{26}$ et, d'une façon de plus en plus marquée inspirent autant les scientifiques que les théologiens. Heller illustre ce processus principalement avec des exemples du domaine de la physique, indiquant, que les succès de la physique liés à l'emploi du concept mathématique de la réalité, évoquent des questions sur l'origine de cette rationalité et sur les sources des aptitudes de compréhension de l'homme ${ }^{27}$. Par contre, les réalisations dans le champs des processus sans retour, dévoilent un aspect „,créatif” de l'évolution ${ }^{28}$. Comme le souligne M. Heller, „La méthodologie propre à la physique moderne nous a appris beaucoup sur les rapports des modèles scientifiques par rapport à la réalité! Si notre connaissance de Dieu est analogique - comme l'affirme la traditionnelle thèse philosophique - pourquoi donc ne pouvons-nous pas intégrer l'analogie de ce nouveau domaine de connaissance; d'autant plus, que de nouveaux «modèles de Dieu» (ce terme s'emploie de plus en plus souvent), comme il s'avère, sont en mesure de rapprocher ces aspects de la réalité divine, devant lesquels les anciennes tentatives restaient impuissantes" ${ }^{29}$. Heller montre que les idées scientifiques peuvent aider dans la formulation de ,nouvelles hypothèses théologiques ou des nouvelles métaphores plus accessibles. Elles peuvent aussi induire l'expérience du mystère («si en physique les choses dépassent tellement notre imagination, quoi dire alors de Dieu?»), ou bien créer un contexte spécifique à une nouvelle re-interprétation et à une nouvelle compréhension de la doctrine des traditions religieuses" ${ }^{\prime 30}$.

Cependant, M. Heller met en garde les théologiens contre une superficielle et unilatérale exploitation des acquis de la science ${ }^{31}$. Il indique aussi l'existence

${ }^{25}$ S. Wszołek, Posłowie. Rozum i wiara. Status questionis u progu trzeciego tysiaclecia, en: S. Wszołek (ed.), Refleksje na rozdrożu, p. 369-370.

${ }^{26}$ Voir M. Heller, O teologicznych interpretacjach fizycznych teorii stworzenia, p. 197-198.

27 Voir M. Heller, O teologicznych interpretacjach fizycznych teorii stworzenia.

${ }^{28}$ M. Heller, Nowa fizyka i nowa teologia, p. 92-95.

${ }^{29}$ M. Heller, Nowa fizyka i nowa teologia.

${ }^{30}$ M. Heller, O teologicznych interpretacjach fizycznych teorii, p. 197-198.

31 „Les chrétiens désirant obtenir de la science une plus profonde compréhension de la création devraient éviter de se limiter aux modèles ou théories cosmogoniques particuliers, mais plutôt se 
d'interprétations erronées des théories scientifiques. Il s'applique à l'analyse de la pertinence des interprétations théologiques des résultats acquis par la science. M. Heller avoue aussi que devant plusieurs problèmes, la science se sent impuissante. Sa division en de nombreuses spécialisations, crée la nécessité d'interpréter les résultas par la philosophie qui pourrait, d'une façon plus large, collaborer avec la théologie. Un facteur important à favoriser l'élaboration d'une synthèse philosophique serait, selon lui, la coopération des scientifiques avec les philosophes: „«l'entrée» des scientifiques professionnels dans la philosophie ouvrirait de nouvelles possibilités. L'enjeu n'est pas seulement de reconnaître que les gens possédant un grand potentiel de connaissance du monde. Ils sont capables souvent de regarder de manière originale les vieux problèmes et d'en apercevoir parfois des aspects inattendus, qui jusqu'a présent échappaient à toute vigilance (on pourrait donner beaucoup d'exemples d'une telle clairvoyance). Mais avant tout, le fait que parfois ils initient des voies tout à fait nouvelles de la recherche philosophique). Sous nos yeux naissent de nouveaux problèmes, provenant des théories scientifiques modernes, dont nous commençons seulement de comprendre l'importance philosophique (ex. l'évolution et la formation des structures dans les états éloignés de l'équilibre, le domaine de l'intelligence artificielle, le rôle de la stabilité structurale dans la philosophie de l'enseignement etc.). Cette soif du monde scientifique de la philosophie entraîne l'accroissement de son intérêt pour la religion et la théologie" ${ }^{32}$.

\section{Philosophie et religion}

La pensée moderne, concentrée sur la vision scientifique et technique de la réalité, rétrécit notre perception humaine. Pour parer cet écueil, il serait nécessaire de faire appel à une réflexion philosophique générale, aussi bien en ce qui concerne la réalité qui nous entoure, que les phénomènes culturels et les fondements de la religion. Ces sujets sont soulevés par J. Tischner et K. Tarnowski.

Ils trouvent que le problème apparaît déjà au niveau de la réflexion sur les bases des relations mutuelles entre la réflexion rationnelle et la foi: „,comment

baser sur les principes les plus fondamentaux de la science comme telle". M. Heller, O teologicznych interpretacjach fizycznych teorii, p. 199.

${ }^{32}$ M. Heller, Nowa fizyka i nowa teologia, p. 88. 
penser l'Absolu de Dieu, sans Le transformer en un objet?"33. La réflexion sur la foi n'est pas facile. Elle ne peut pas faire abstraction du fait que croire en Dieu implique aussi une référence à des croyances et à des modalités de croire. Le croyant est contraint à répondre à un certain nombre de questions essentielles. Quelle est l'origine de ma foi ? Qu'est-ce-que cela veut dire, ,je crois"? Comment chercher la vérité concernant la foi et que signifie ,recherche de la vérité"? S'agit-il de croire en quelqu'un, ou bien de croire ce que quelqu'un professe $?^{34}$ On ne peut pas trouver de réponse à ces questions sans amorcer de réelles réflexions sur l'homme.

J. Tischner prend comme point de départ le fait que la foi n'apparaît pas comme une conséquence d'un raisonnement; ,elle apparaît d'elle-même, conformément à ses lois propres. La foi est originelle et la réflexion sur la foi se développe après coup. La philosophie et la religion, bien entendu peuvent décrire la foi. Elles sont en mesure de montrer à quel point la foi est d'un autre ordre que toute théorie sur la foi" ${ }^{35}$. Il démontre également qu'un raisonnement religieux ne peut pas se concentrer sur des phénomènes observables et intersubjectives concernant Dieu. En suivant cette voie on devrait toujours faire appel aux courants philosophiques basés sur la pensée objective, intentionnelle et monologique. Ces courants, fortement liés à la pensée scientifique, se reportent aux expériences communément accessibles, ${ }^{36}$ mais ils omettent les expériences du vécu religieux, essentielles pour la constitution de la foi, pourtant invérifiables par une analyse intersubjective. „Il ne faut pas rechercher une parenté entre Dieu et la créature, surtout pas sur le terrain de l'organisation technique de l'existence, mais bien sur le terrain des liens interpersonnels" ${ }^{37}$. Si c'est ainsi, alors „il faut quitter l'ontologie, indépendamment des paradoxes qui en résulteront" 38 et reconnaître que „la condition subjective de la possibilité d'un résonnement religieux est le Moi

${ }^{33}$ K. Tarnowski, Ustyszeć niewidzialne. Zarys filozofii wiary, Kraków 2005, Instytut Myśli Józefa Tischnera, p. 406.

${ }^{34}$ Ces questions nous renvoient à d'autres questions: „Que signifie-t-il que la foi recherche la compréhension? Comment se fait-il que la foi ne se suffit pas à elle-même mais qu'elle sollicite les lumières de la raison? Et plus loin: que signifie-t-il que la raison recherche la foi? Comment se fait-il que la raison ne se suffit pas à elle-même, mais qu'elle cherche un compromis avec la foi?". J. Tischner, Wokól spraw wiary i rozumu, p. 15.

${ }^{35} \mathrm{~J}$. Tischner, Wokót spraw wiary i rozumu, p. 27-28.

${ }^{36} \mathrm{~J}$. Tischner, Myślenie wedtug wartości, p. 354-355.

${ }^{37}$ J. Tischner, Świat ludzkiej nadziei, Kraków 2000, Znak, p. 214.

38 J. Tischner, Filozofia dramatu. Wprowadzenie, Kraków 1999, Znak, p. 263. 
axiologique. Le Moi qui, dans le choix de la vérité et sa requête, a retrouvé la justification de sa valeur" ${ }^{\prime 3}$. Toutefois, le Moi axiologique ne suffit pas en tant que tel dans la quête de la vérité. „La condition nécessaire pour obtenir le savoir fondé sur un sujet, c'est une affirmation d'un témoin fiable". C'est pourquoi le Moi doit s'appuyer sur un résonnement dialogique - l'élément essentiel de la pensée religieuse. „L'expression d'une présence de la pensée dialogique au sein de la pensée religieuse est le principe «fides quaerens intellectum» - la foi cherchant la compréhension, et plus précisément la compréhension spécifique d'Augustin (...) : «fides» s'associe à «fidelitas», qui signifie fidélité. «Fides» n'est pas «une foi en quelque chose», mais «la foi en quelqu'un», plus exactement la fidélité envers quelqu'un" ${ }^{\prime 40}$. La fidélité s'associe étroitement à la foi en quelqu'un, mais, comme l'affirme Tischner - la disposition à avoir foi est une attitude naturelle de l'esprit ${ }^{41}$.

Un témoin fiable montre à l'homme que sa quête de comprendre des perspectives nouvelles, le renforce, le fortifie et le libère de nombreuses illusions, méprises, fausses appréciations et auto-évaluations ${ }^{42}$. Le dialogue avec le témoin fiable fait découvrir la vérité et mène l'homme vers le Bien. Il fait entrevoir une vérité simple, notamment que tout mensonge est une contradiction de la raison, une négation du sens de l'humanité, menant vers le Mal ${ }^{43}$. Dans cet appel à la communication ,dialogique” et à l'ouverture vers l'autre, nous trouvons les échos de la pensée de M. Buber ${ }^{44}$. Sa nostalgie du „vrai dialogue, d'une certitude avec une autre certitude, mais aussi d'une personne ouverte à une autre personne réciproquement ouverte”. Grâce à ceci ,il se créera une réelle communauté" 45 .

${ }^{39} \mathrm{~J}$. Tischner, Myślenie według wartości, p. 347-348.

40 J. Tischner, Myślenie wedlug wartości.

${ }^{41}$ Voir J. Tischner, Myślenie wedtug wartości, p. 349-350. „La croyance d'un homme envers un autre homme se comprend d'elle-même, avant même que la raison réflexive le comprenne. La raison n'est pas une chose étrangère et artificielle face aux differentes fois, mais reste sa réalité intérieure et cachée. La foi, d'elle-même, sollicite la compréhension. Elle attire la raison comme le semblable attire le semblable. La raison qui entre au fond de la fidelitas est surprise car ce qui lui paraissait originairement étranger, ou même hostile, se devoile être ... elle-même. Le principe de croire l'autre homme est un principe propre de la raison". J. Tischner, Wokól spraw wiary i rozumu, p. 18.

${ }^{42}$ J. Tischner, Myślenie wedtug wartości, p. 351-352.

${ }^{43}$ Voir J. Tischner, Filozofia dramatu, p. 189-190.

${ }^{44}$ Voir M. Buber, Ja i Ty, traduc. polonais J. Doktor, Warszawa 1992, IW PAX, p. 146, voir aussi pp. 214-225, 235, 236-237.

${ }^{45}$ M. Buber, Ja i Ty, p. 214. 
J. Tischner, avec une obstination digne d'admiration, démontre combien le chemin d'un chercheur de la vérité peut être changeant et long, quand il ne se laisse pas tenter par des raccourcis ontologiques et des simplifications méthodologiques. Durant son évolution, le personnalisme est soumis à la critique et mis en parallèle avec le thomisme, quand il essaie d'absolutiser ses conclusions. „Celui qui fait de Dieu le coeur de l'être, peut égarer l'homme; celui qui fait de l'homme le centre, peut égarer Dieu. (...) Quand Dieu parle à l'homme, et quand l'homme parle à Dieu, le problème du centrisme disparaît"46. Dans un dialogue du cœur, personne ne devrait se placer au centre. Dans le cas d'un Dieu ardent, aimant moi et l'Autre; et moi aimant Dieu et l'Autre, nous existons pour nous et avec nous. Nous nous comprenons, nous ressentissons notre présence et le bien qui réside en nous, attendant les conditions favorables pour son éveil. Nous avançons vers un degré supérieur de la maturité de conscience - nous domptons l'orgueil intellectuel, l'égoïsme; nous récupèrerons la puissance d'agir, renforcée par la certitude de la vérité acquise et la foi dans le sens de notre propre action. Avec de tels fondements on peut débattre avec humilité, patience et sérénité des questions philosophiques et théologiques spécifiques. Les problèmes qui apparaissent maintenant dans la perspective de l'éternité récemment retrouvée, de la liberté sans limites, de l'amour et de la vérité pénétrant tout, perdent de leur importance et leur considération devient plus facile pour un esprit, qui a retrouvé le calme et la paix de l'âme.

L'essentiel de la philosophie de J. Tischner est la conviction que la possibilité de communiquer avec un autre homme, est réalisée grâce à la liberté, qui est un don de $\mathrm{Dieu}^{47}$. Ce n'est qu'alors que nous allons pleinement comprendre Sa sensibilité pour l'autre homme et Son manque d'acceptation des vérités établies depuis les siècles et pour un avenir illimité. Dieu a doté ma conscience des empreintes de son Infinité. Grâce à la réflexion je retrouve en moi les désirs qui sont l'expression de „la nostalgie absolue”. „«Le désir» se tourne vers ce qui est infini". ${ }^{48}$ Et bien que je ne sache toucher ni déterminer convenablement ce que je désire, toutefois je peux nommer et déterminer ma nostalgie et par là, l'espoir et l'amour. Je peux déterminer ce que j'attends des autres et ce que je

${ }^{46}$ J. Tischner, Myślenie wedtug wartości, p. 195.

47 „La foi est une réponse au don. Le don est un trésor. Ce trésor c'est l'autre et je m’y retrouve seulement à la deuxième place. Pour cela les hommes qui ont le désert dans l'âme ont du mal à trouver Dieu. Ils n'ont rien à offrir à Dieu sinon ... leur propre égoïsme”. J. Tischner, Przekonać Pana Boga, Kraków 1999, Znak, p. 21.

48 J. Tischner, Spór o istnienie człowieka, p. 329. 
voudrais leur offrir en retour pour ce qu'ils m'ont déjà donné. Les principes de la religion sont formulés à travers de tels traits négatifs, religion qui pourtant „n'est pas engendrée en imposant les entraves”. Selon J. Tischner „La religion est une manifestation des liens déjà existants, quoique inconscients"49; des liens qui ont été déterminés conjointement par l'enracinement dans notre conscience et par notre conscience du Bien infini. Cette religion rappelle que l'homme est connecté à la vie de Dieu, et devient une partie de la nature divine. En recevant le don divin il doit rendre aux Autres sa dette de reconnaissance. Dieu est présent dans notre vie, il se révèle dans l'histoire, nous le retrouvons dans des situations concrètes et dans les rencontres singulières. Il nous devient proche, nous reconnaissons $\mathrm{Sa}$ face, et grâce à ceci nous commençons à comprendre non seulement qui nous sommes, mais aussi pourquoi nous existons.

$\mathrm{K}$. Tarnowski évolue dans la sphère des problèmes semblables. Il se concentre, cependant, sur l'observation qu'on ne peut pas s'approprier certaines vérités. En plus, juste au moment où nous avons l'impression de les posséder, elles se retournent contre nous - s'averrant être des notions sans valeur ou devenir des théories stériles.

En se référant aux structures fondamentales de la foi, il observe beaucoup de pièges où la pensée peut s'enliser. Tout d'abord, nous adaptons trop souvent l'objet de notre foi - Dieu - à nos possibilités modestes de connaissance. Nous Le remplaçons alors par une notion abstraite qui nous cache ce qui constitue l'essentiel de la divinité de Dieu. Tarnowski indique qu'une telle simplification déforme aussi bien la pensée sur la foi que la foi, elle-même. Conscient des connotations historiques affectant des notions comme l'être, l'absolu, la transcendance ou le bien, Tarnowski observe attentivement dans quelles limites et sous quelles conditions nous pouvons nous en servir en parlant de Dieu. Il évoque, que l'objet de la philosophie de la foi est tellement fragile, qu'il demande plutôt une méditation que des théories expéditives. Saisir la foi par la raison n'est possible qu'à travers une expérience concrète et non par un modèle théorique. Le philosophe se concentre donc sur l'experience religieuse originelle, à laquelle il donne la notion d'une „foi fondamentale”.

La foi fondamentale est une ouverture primaire, c'est la condition du sujet de se reporter vers autre chose: ,elle constitue la racine même des conditions des possibilités d'apparition des phénomènes et du sujet à lui-même. Les phénomènes apparaissent pour la conscience humaine, qui reçoit les diverses qualités arrivant

\footnotetext{
49 A. Michnik, J. Tischner, J. Żakowski, Między Panem a Plebanem, Kraków 1995, Znak, p. 288.
} 
grâce à la confiance, liée indissociablement à un pré-événement de la vie comme «mienne»" ${ }^{50}$. La confiance renvoie à quelque chose, elle indique quelque chose de plus. „La foi fondamentale n'est rien, si elle n'aspire pas «en dehors» et à la fois «en profondeur»; et donc peut être en dehors du temporaire vers l'éternité. Et elle ne pressent pas le bien infini comme une réalité impossible à saisir, à comprendre et même, au sens ordinaire du terme, à identifier, mais dans laquelle à travers la liberté elle peut participer" ${ }^{\text {"51 }}$. La foi fondamentale ouvre l'homme à la perspective de la foi, quoiqu'elle n'y conduise pas toujours. Elle est cette experience qui „ne permet pas de fermer l'horizon par un schéma de la totalité, comprise de manière quelconque. Elle le laisse constamment ouvert par l'intermédiaire des schémas du bien infini" ${ }^{52}$. Elle ne se soumet pas aux schémas par ce „qu, elle contient «un surplus» inépuisable de la raison” ${ }^{53}$. Ce surplus obtient une dimension religieuse tout à fait nouvelle, uniquement quand „le sujet de la foi fondamentale découvre qu'il a été invité par un témoin digne de foi - à se dépasser lui même - et à dialoguer avec le caché" ${ }^{54}$.

La foi fondamentale se transforme en une foi religieuse quand l'homme aperçoit l'Autre sur son chemin. Dans la philosophie de Tarnowski, la Différence est une notion non précisée - il me semble - avec préméditation. „Cette Différence - ceci est primordiale - ne peut pas être raisonnée, mais on peut la désirer" ${ }^{\prime 55}$. La Différence se rapporte à l'Autre, qui est affirmé dans l'acte de la foi, mais aussi à l'Autre, qui ne peut en quelque sorte qu'effleurer - et que je découvre en moi. Je retrouve l'Autre par delà les émotions quotidiennes, par les réflexions et les prières. Grâce à ceci l'Autre me devient familier, plus Proche, il fait partie de moi-même, il est présent en moi. Ce n'est que par cette voie que l'autre ne se transformera en Etranger.

La recherche du philosophe qui s'occupe des questions de religion serait, comme le suggère Tarnowski, d'examiner des limites de l'expression de l'inexprimable et la protection de la sensibilité religieuse contre les appétits des théologiens armés en schémas religieux. Le philosophe ne peut pas décrire ce que signifie l'Autre, mais il peut seulement indiquer ce que signifie pour moi

\footnotetext{
${ }^{50} \mathrm{~K}$. Tarnowski, Ustyszeć niewidzialne. Zarys filozofii wiary, p. 441.

${ }^{51} \mathrm{~K}$. Tarnowski, Ustyszeć niewidzialne. Zarys filozofii wiary, p. 447.

${ }^{52} \mathrm{~K}$. Tarnowski, Ustyszeć niewidzialne. Zarys filozofii wiary.

${ }^{53} \mathrm{~K}$. Tarnowski, Ustyszeć niewidzialne. Zarys filozofii wiary.

${ }^{54} \mathrm{~K}$. Tarnowski, Ustyszeć niewidzialne. Zarys filozofii wiary, p. 448.

${ }^{55} \mathrm{~K}$. Tarnowski, Ustyszeć niewidzialne. Zarys filozofii wiary, p. 438.
} 
la présence de l'Autre et quelles sont les conséquences pour moi et dans ma vie, de cette Différence tellement proche.

Les idées de Tarnowski, autant que celles de Tischner indiquent que dans une vie religieuse deux univers s'unissent. L'univers de l'homme et l'univers du témoignage de l'espoir humain ${ }^{56}$. La philosophie qui s'occupe de ce vécu devrait focaliser ses recherches sur l'homme et sur les besoins qu'il éprouve, pour dépasser les limites lors de sa quête du sens de tout ce qui est tangible et de tout ce qui dépasse la réalité. Evidemment, elle doit le faire avec humilité, consciente de ses propres limites et de l'impossibilité d'exprimer en mots les définitions et les théories relatives aux aspects de notre réalité, tant extérieure que spirituelle.

Sur cette base, on pourrait „revenir vers les anciennes métaphores et les symboles, redécouvrir leur sens, et essayer de réfléchir à partir de ces images. On pourrait ainsi s'élever au dessus des contradictions de nombreuses théories et concepts, pour arriver à l'essentiel"57. Cette possibilité de concilier la foi et la raison peut paraître à certains comme insuffisante ou trop prudente. Mais c'est une tentative sincère de déterminer les conditions acceptables d'inspiration, d'exploration et d'ingérence mutuelle de ces domaines autonomes. Certes, même en étant prudent, des possibilités de pénétration entre ces deux domaines peuvent rester inexploitées. Les philosophes peuvent se tromper; les théologiens, les directeurs de conscience et les croyants peuvent perdre de vue ce qui importe. Tous ceux, qui pourraient et qui voudraient entreprendre la réflexion et le dialogue sur les plus profondes relations entre la foi et la raison, peuvent se tromper.

Or, un approchement de ces deux domaines n'est pas possible sans la présence des hommes de relais, dits «pontifes» (,faiseur de ponts”) - qui se consacrent avec passion tant à la science, qu'à la philosophie et qui sont „plongés” dans la foi. Ceci concerne tant les laïcs scientifiques, sensibles aux questions de la foi, que „les hommes d'Eglise”, qui étudient les domaines de la pensée moderne. Les prêtres pourraient et même devraient être des „hommes de relais plus importants"; ils sont les „,bergers de l'âme”, sous réserve cependant qu'ils soient bien préparés à faire front aux exigences du temps moderne, de ses défis, de ses découvertes, de ses réalisations, mais aussi des adaptations. Sur de tels

\footnotetext{
${ }^{56}$ Voir K. Tarnowski, Józefa Tischnera myślenie wokó zbawienia, p. 45-56.

57 J. Tischner, Myślenie wedtug wartości, p. 360.
} 
The Person and the Challenges
Volume 5 (2015) Number 1, p. 23-40

intermédiaires repose le poids réel de la transformation de la foi dépassée et aveugle en une foi vécue, profonde, facilement comprise, et qui reste à l'écoute.

Il faut être conscient qu'une popularisation du débat sur les relations mutuelles entre la foi et la raison posera aux philosophes et aux théologiens beaucoup de problèmes nouveaux. Mais sûrement cela ne va pas faire du mal ni à la foi, ni à la raison. Cela ne n'affectera pas non plus ceux qui vont entreprendre l'effort de méditer sur la foi et la croyance éclaircie par une compréhension de plus en plus profonde de leurs convictions.

\section{Bibliography}

Buber M., Ja i Ty, traduc. polonais J. Doktor, Warszawa 1992, IW PAX.

Heller M., Czy fizyka jest nauka humanistyczna??, Tarnów 1998, Biblos.

Heller M., Nowa fizyka i nowa teologia, Tarnów 1992, Biblos.

Heller M., O teologicznych interpretacjach fizycznych teorii stworzenia, en: StwórcaWszechświat - Człowiek. Wybór tekstów wygłoszonych podczas konferencji z cyklu Boże działanie w perspektywie nauki zorganizowanego przez Watykańskie Obserwatorium Astronomiczne (Specola Vaticana) i Center for Theology and the Natural Sciences (CTNS; Berkeley, California): 1988-2001, 1 (2006), p. 186-199.

Jean-Paul II, Encyclique, Fides et Ratio.

Kłoczowski J.A., Leszka Kołakowskiego myślenie o religii, en: Polacy o religii. Od myślenia religijnego do filozofii, J. Barcik, G. Chrzanowski (ed.), Kraków 2005, Wydawnictwo Naukowe PAT.

Michnik A., Tischner J., Żakowski J., Między Panem a Plebanem, Kraków 1995, Znak.

Tarnowski K., Ustyszeć niewidzialne. Zarys filozofii wiary, Kraków 2005, Instytut Myśli Józefa Tischnera.

Tischner J., Filozofia dramatu, Kraków 1999, Znak.

Tischner J., Myślenie wedtug wartości, Kraków 1982, Znak.

Tischner J., Przekonać Pana Boga, Kraków 1999, Znak.

Tischner J., Świat ludzkiej nadziei, Kraków 2000, Znak.

Tischner J., Wokół spraw wiary i rozumu, en: S. Wszołek (ed.), Refleksje na rozdrożu, Wybór tekstów z pogranicza wiedzy $i$ wiary, Tarnów 2000, Biblos.

Wszołek S., Postanie Jana Pawła II do George'a Coyne'a na tle encykliki Fides et Ratio, en: J. Mączka (ed.), Wiara i nauka. Materiały z sesji naukowej i dyskusji panelowej, Kraków 2010, Wydawnictwo UJ. 